\title{
Development of Reasoning Habits Through Lesson Study and Open Approach Teaching Practices
}

\author{
Thong-oon Manmai ${ }^{1}$, Maitree Inprasitha ${ }^{1}$, Narumon Changsri $^{1} \&$ Auijit Pattanajak ${ }^{2}$ \\ ${ }^{1}$ Faculty of Education, Khon Kaen University, Thailand \\ ${ }^{2}$ Institute for Research and Development in Teaching Profession for ASEAN, Khon Kaen University, Thailand \\ Correspondence: Narumon Changsri, Faculty of Education, Khon Kaen University, Khon Kaen/40002, Thailand. \\ Tel: 66-911-305-256. E-mail: changsri_crme ${ }^{\circledR}$ kku.ac.th
}

Received: June 06, 2020; Accepted: June 20, 2020; Published: June 22, 2020

The research is supported by the Centre for Research in Mathematics Education (CRME), Khon Kaen University, Khon Kaen 40002, Thailand.

\begin{abstract}
This research aimed to investigate the development of reasoning habits after introducing the lesson study and open approach to teaching practices. A total of eight $6^{\text {th }}$-Grade students from Chumchon Ban Kaeng Khro Nong Phai School, Chaiyaphum province, Thailand were purposively selected to participate in this research. This is because they have attended the mathematics lessons using the innovation of lesson study and open approach. A qualitative research design was employed using five types of research instruments, namely 13 lesson plans, observation records, field notes, interview protocol, and students' worksheets. Data were analyzed using content analysis and categorizing the students' reasoning habits according to the National Council of Teachers of Mathematics' framework. The results revealed that students can analyze a problem situation by making reasoning conjectures, applying previously learned concepts, seeking patterns and relationships as well as making representations. Besides, they can also implement a strategy by using mathematical operations to solve problems. Moreover, students can seek and use connections to discover the linkage between how to solve the problems differently and identify the relationship of the approach used to get the same answer. Finally, the students can reflect on a solution as they can find different ways to solve the problems after considering the reasonableness of a solution, interpret a solution, justifying reasons to support or validate how to solve the problems, as well as generalize a solution. The overall results are successfully contributed to the importance of lesson study and open approach teaching practices in terms of enhancing $6^{\text {th }}$-Grade students' reasoning habits.
\end{abstract}

Keywords: lesson study, open approach, reasoning habits

\section{Introduction}

Previous research has investigated mathematical subject knowledge and teachers' belief during the implementation of a practical mathematics scheme engaging them in new pedagogical knowledge (Boyd \& Ash, 2018). Mathematical reasoning allows students to solve a mathematical problem without algorithms, or a set process. The best way to develop mathematical reasoning habits of students is by giving them opportunities to solve a problem in a variety of ways (Mathnasium of Littleton, 2016). There is no place other than the classroom where mathematics looks like a bunch of problems on a sheet of paper (Mathnasium of Littleton, 2016). As a result, learning to use and apply mathematical reasoning to problems takes more time if comparing to the exploration typically given in the classroom as indicated by Steffee and Thompson's (2000) study. This is because the mathematical reasoning learning process requires students to think and wrestle with concepts, which may cause some discomfort at first, especially if they are used to figuring out answers quickly. Students also possess mathematical reasoning by thinking about and describing mathematical strategies and why certain strategies are effective and others are not. The process of describing their thought requires analysis of the mathematical concepts (Oslington, Mulligan, \& Van Bergen, 2020).

Lesson Study (LS) is a teacher professional development model originated from Japan and LS is considered as teacher-led professional learning (Tan, Lim, \& Chew, 2017). According to Wang-Iverson \& Yoshida (2005), in the LS process, a group of teachers plan, conduct, and reflect on research lessons collaboratively and systematically. 
In other words, a research lesson refers to the lesson taught by a teacher to a group of students, in accordance with the lesson plan developed by the LS group. During the research lesson, other members of the LS group observe and collect the data of the students' learning during the lesson. Reflection session was conducted after the research lesson, where the members of the LS group reflect based on the data they have collected during the research lesson (Tan et al., 2017). In short, LS allows teachers to collaborate to design, test, and improve lesson arrangements that are probably to improve learning and be useable by other teachers (Tall, 2008). Thus teachers have a means for planning, observing, and conferring with others (Meyer \& Wilkerson, 2011). In recent years, the mathematics education community particularly has utilized substantially LS in teaching practices. The LS concept was adopted by Inprasitha (2011) in Thailand and the LS process consists of three important steps, namely collaboratively plan, do, and see.

Open Approach (OA) refers to a teaching approach that is concentrated on allocating with an open-ended problem, which is projected to improve the classroom instruction effectively (Becker \& Shimada, 1997). In this line of reasoning, teaching by using the OA method aims that all students can learn mathematics in response to their mathematical power, escorting with a certain degree of self-determination of their learning, and can elaborate the quality of their process and outcomes toward mathematics (Nohda, 2000). In short, teachers who employ OA in their teaching need to understand their students' ideas as much as possible, to sophisticate the ideas in mathematical activities by utilizing students' negotiations with others and/or teachers' advice, and to encourage their selfregulate in elaborating the learning activity mathematically.

According to Kaur, Awang Hashim, \& Noman (2014), students are not given sufficient exposure to real-life learning and hands-on experiences in Thai schools. Therefore, Thai mathematics classroom is usually structured, depend on rote memorization skills, and have a minimum class discussion. Eddy and Inprasitha (2012) proposed the four steps of OA, namely posing open-ended problems, students' self-learning, whole-class discussion and comparison, and summing up by connecting students' mathematical ideas which could be overcome the problems of teaching mathematics in Thailand that is emphasizing on the content transfers, introducing formulas and rules, and repeated the exercise for enhancing memorization, as mentioned by Inprasitha (2014) and Kaur et al. (2014). With the combination of these two innovations, LS and OA, mathematics teachers have more opportunity to assess students' reasoning learning abilities (Inprasitha, 2011). Currently, LS and OA innovations are one of the most respected forms of teaching practices and is one of the factors that have resulted in the success of the basic school system in Thailand. In this line of reasoning, this research was designed to investigate the development of reasoning habits after implementing the LS and OA innovations to teaching practices.

\section{Method}

\subsection{Research Samples}

A total of eight students were purposively selected because they had involved in the School of Professional Development of Mathematics Teachers Project using the innovation of LS and OA which introduced by the Center for Research in Mathematics Education (CRME), Faculty of Education, Khon Kaen University since 2003. The participants were at their Grade Six basic education at Chumchon Ban Kaeng Khro Nong Phai School, Chaiyaphum province, Thailand

\subsection{Research Design, Instruments and Data Collection}

In this study, a qualitative research design was employed to convey meaning via a detailed description. Qualitative data were collected through various sources, namely lesson plans, observation records, field notes, interview protocol, and students' worksheets. The artifacts collected included classroom observation, field notes, lesson plans, students' worksheets, and teaching materials used during the 13 research lessons. Besides, researchers also interviewed the eight participants individually to explore their views on their actions and behavior during the research lessons. An interview protocol was used as an instrument. All the learning observation and interview sessions were video and audio recorded, followed by verbatim transcription. A qualitative design is considered the most suitable and powerful method to explore educational issues in this research, that requires a comprehensive and complex social setting, and the meaning that participants showed within those setting to justify reasoning habits of the participants. A case study was employed as it has an opportunity to bend several methods into justified the research design.

\subsection{Research Procedures}

The LS cycle consists of three steps, namely 'Plan', 'Do', and 'See' (Inprasitha, 2011) was employed as a research procedure. An LS group consisting of researchers and teachers who prepared and designed collaboratively the 13 lesson plans with each plan needing 60 minutes to implement as the first step of the LS cycle. LS group members 
determined open-ended mathematics problems and learning activities to incorporate with $\mathrm{OA}$ in the research lesson plans so that students would engage with reasoning habits during the research lessons. This is followed by the second step by conducting the research lesson. All the LS group members observed the research lesson implementation. They filled in the field notes when they were observing. In the second step, researchers focused on the categories of students' reasoning habits to collect relevant data. After the research lesson, all the LS group members gathered and made their reflection as the final step of the LS cycle. The LS group members discussed and did reflections as well as investigated the categories of reasoning habits from various sources that they obtained during the research lessons. The final step was performed once a week.

The OA consists of four steps, namely 'posing open-ended problem', 'students' self-learning', 'whole-class discussion and comparison' and 'summarize through connecting students' mathematical ideas emerged in the classroom' (Inprasitha, 2011) were incorporated into the 13 research lesson plans. The first step is 'posing openended problem' which refers teacher inserts problem situations as an assignment or worksheet to provide an opportunity for students to face the problems until doing the task-based learning activities. Next, the 'students' self-learning' step refers to the learning process whereby students perform their reasoning habits by explaining, writing, doing worksheets, and speaking for expression together with their peers. The third step is the 'whole-class discussion and comparison' which is the process that students present their discussion outcomes derived from the second step by explaining, writing, and doing worksheets as the additional identification of students' reasoning habits. The final step of OA is 'summarize through connecting students' mathematical ideas emerged in the classroom', meaning teacher summarizes the lesson based on students' conceptual understanding derived from their worksheets as well as their presentation outcomes. Figure 1 shows the conceptual framework of this research by incorporating LS and OA.

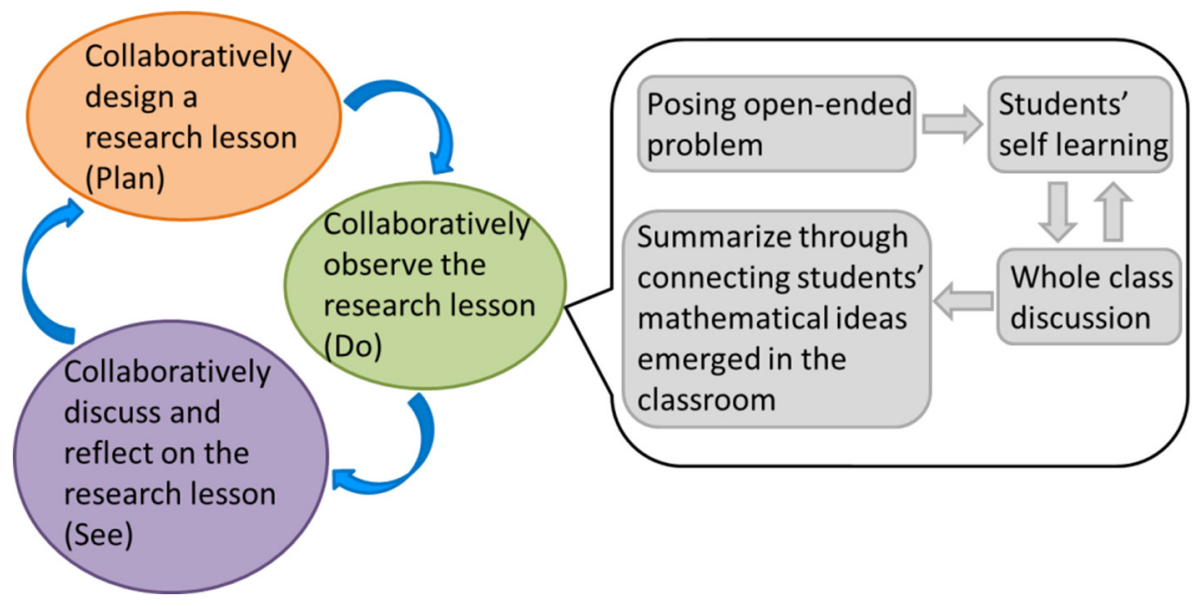

Figure 1. Conceptual Framework

\subsection{Data Analysis and Triangulation}

All the qualitative data collected from various sources encompassing lesson plans, field notes, interviews, observation, students' worksheets were transcribed verbatim, and then divided into categories. A category means the part of the transcript which was related to a theme of reasoning habits. The categories were then coded to become the themes of reasoning habits, such as analyzing a problem, implementing a strategy, seeking and using connections, and reflecting on a solution. These categories were coded based on the National Council of Teachers of Mathematics (NCTM)'s (2009) framework. The findings of all research lessons were triangulated with field notes, lesson plans, students' worksheets, and interview data. Later, the coding was compared across the 13 LS cycles to explore any changes that occurred.

\section{Results}

The results of this research are presented by following the framework of NCTM (2009) with four categories after utilizing LS and OA research lessons on the topic of 'Volume'. The problem situation was to be investigated:

"If you want to build a box with a volume of 200 cubic centimeters with width, length, and height how are you going to calculate its volume?" 


\subsection{Category 1: Analyzing a Problem}

The results revealed that students can analyze the problem situation that their teacher assigned to them. This reflects in their learning process whereby they can represent the concepts, apply previously learned concepts, seek patterns and relationships, and create conjectures in the classroom. The first evidence was derived from observation's artifact Item 36 stated that "What this assignment is? Shall we read this question again?" This statement reflects that students tried to understand the problem situation. Besides, observation's artifact Item 39, the student mentioned that "Well, shall we do the factorization? Do we?" and Item 41 who did the representation method with factorization as the method to solve the problem situation. There was one student represented the factorization as a conjecture to the group members to solve this problem.

Figure 2 shows results obtained from the observations revealed that verbal expressions and representation of the students as elaborated below.

Item 36 Student 1: What this assignment is? Shall we read this question again?

Item 39 Student 2: Well, shall we do the factorization? Do we?

Item 40 Student 1: Can we do it in various methods?

Item 41 Student 2: The first one is factorization (plotting the factor of 200 and explain to the group). First, we will do the factorization of 200, so we have 1 and 200, 2 and 100, 5 and 40, 8 and 25, and 10 and 20 . We will match these factors but we will find the volume. So, these factors will be multiplied with each other, and then multiply them by 1 , like $10 \times 20$ is equal to $200 \times 1=200$.

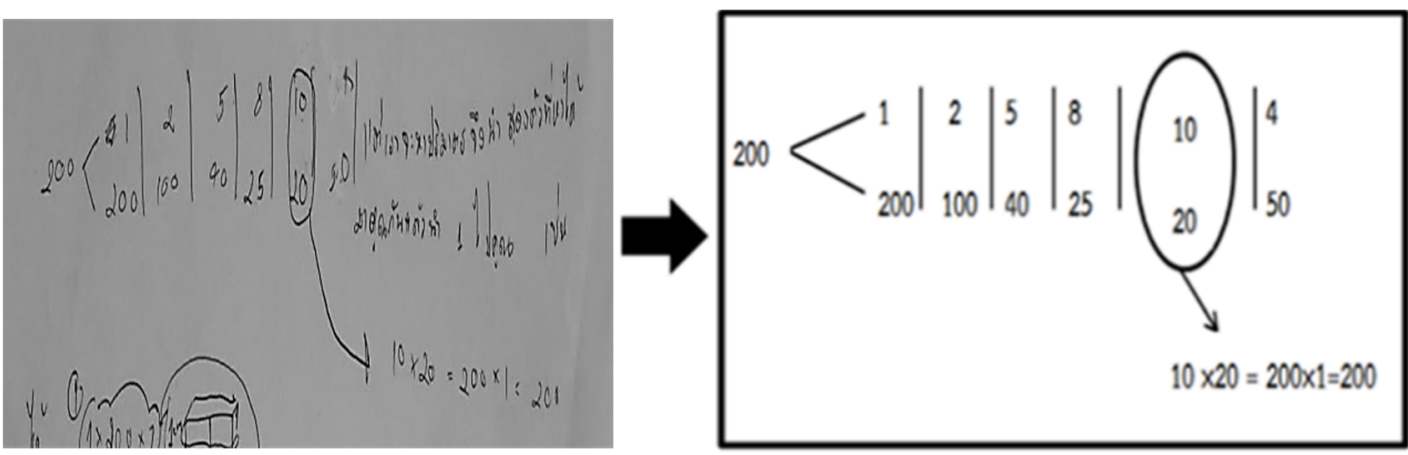

Figure 2. The Students' Representation in Solving the Problem Situation

Besides, observation artifact Item 58 student said "And the other method is... (explaining many methods with writing them on the worksheet as follows)". Figure 3 shows students were able to seek pattern and relationship from 200 cubic centimeters box drawing with a defined width, length, and height, as well as it was a related data of the above factorization modeling

\section{Item 58 Student $2 \quad$ : And the other method is... (explaining many methods with writing them on a} worksheet as follows).
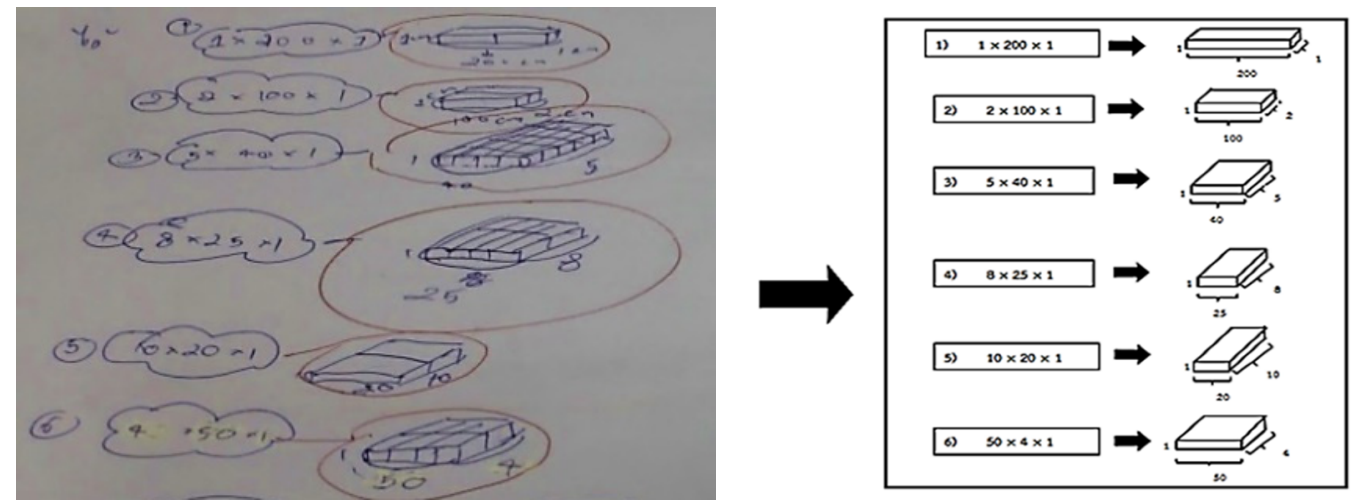

Figure 3. The Students' Seeking Pattern and Relationship 
The above students' representations are pieces of evidence to categorize as 'analyzing a problem' which is the first category of students' mathematical reasoning habit according to NCTM's (2009) framework.

\subsection{Aspect 2: Implementing a Strategy}

The results revealed that students can implement the ways of problem-solving using mathematical operation as elucidated at the following observation artifacts:

$$
\text { Item } 48 \text { Student 2: (Student } 2 \text { plotted the factor of } 200 \text { and explained to the group members). }
$$

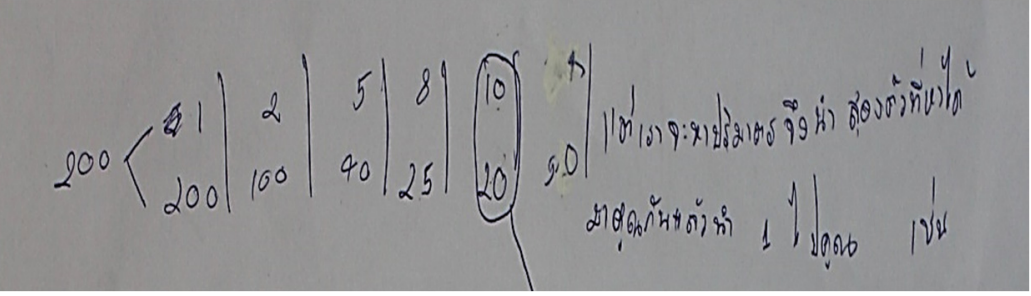

Figure 4. The Students' Factorization as the Problem-solving Strategy

Item 59 Student $2: 200$ cubic centimeters boxes consist of 200 lengths, 1 width, and 1 height (drawing it in the worksheet).

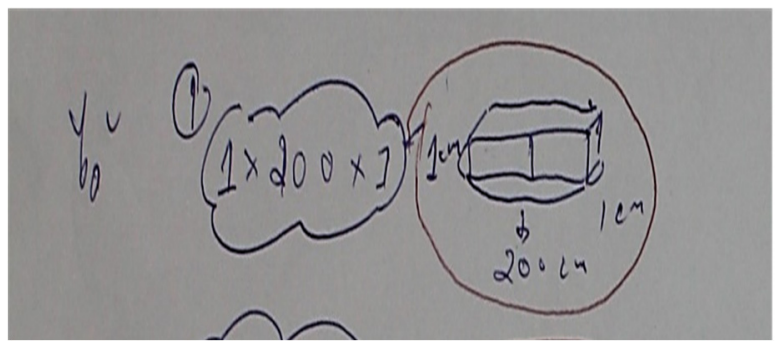

Figure 5. The Students' Problem Solving by Drawing Strategy

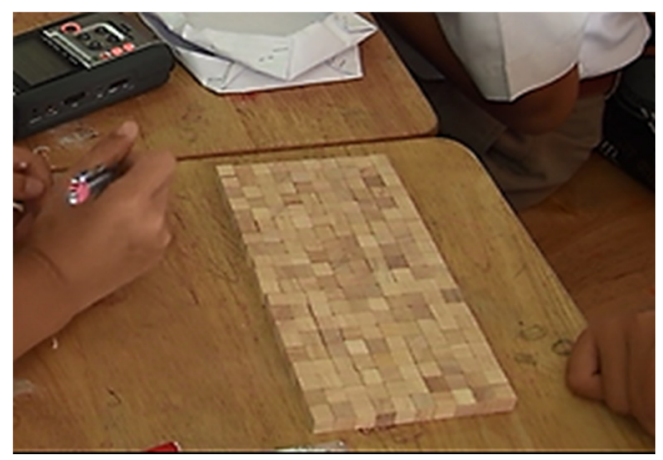

Figure 6. The Woodblock Composition as Students' Problem-solving

There were three shreds of evidence of the second category of reasoning habits identified to be 'implementing a strategy', such as observation artifact Item 48 student applied factorization as the problem-solving strategy, as shown in Figure 4, Item 59 student used the drawing strategy as shown in Figure 5, and Item 199 student composed the woodblock, as the problem-solving strategy, as shown in Figure 6. From these above strategies, students adapted their old knowledge with new situations, and applied mathematical operations, multiplication, and division, to create 200 cubic centimeters boxes. The above students' representations are shreds of evidence to categorize as 'implementing a strategy' which is the second category of students' mathematical reasoning habit according to NCTM’s (2009) framework. 


\subsection{Aspect 3: Seeking and Using Connections}

The results revealed that students can discover linkage between the differences, sorting out the problems, finding the relationship of that approach by getting the same answer as shown in the following observation artifacts:

Item 51 Student $2:$ Here it is, $20 \times 10$ is the factorized factor (showed to the classmates with arrow) So I drew the box picture with length 20 and 10 widths so it was 200 . Oh! It was so many.
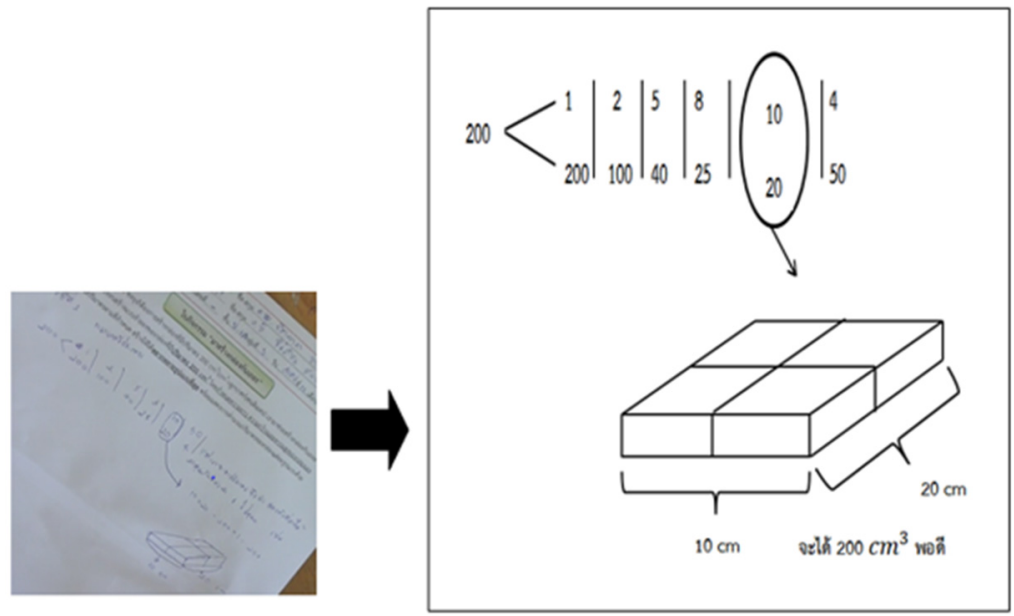

Figure 7. The Students' Seeking and Using the Connection

\begin{tabular}{lll}
\hline Item 69 & Student 1 & : Hey bro! what will you draw here? (pointed to the worksheet). \\
Item 70 & Student 2 & : I will let it extends that.
\end{tabular}

There were three pieces of evidence of the third category of reasoning habits identified to be 'seeking and using connections', such as observation artifact Item 51, a student said "Here it is, 20x10 is the factorized factor (showed to the classmates with arrow)" So I drew the box picture with length 20 and 10 widths so it was 200. Oh! It was so many. As indicated in Figure 7, Student 2 tried to explain to her group members about the relation between her drawing box and factorization. During the relation between the drawing box and factorization, she found that the problem-solving situations, including Item 70 Student 2 replied to her friends "I will let it extends that", this mentioned message approved that she was able to apply old problem-solving method to magnify or revise with the other problems. This process is considered as the third category of students' mathematical reasoning habit, namely 'seeking and using connection' according to NCTM's (2009) framework.

\subsection{Aspect 4: Reflecting on a Solution}

The results revealed that students can find different ways to consider the reasonableness of the solution, interpret the solution, justify or validate, and generalize the solution as shown in the following observation artifacts:

\begin{tabular}{|c|c|c|}
\hline Item 124 & Teacher & : The first one was factorization, so what will you do next? \\
\hline Item 125 & Student 2 & $\begin{array}{l}\text { : Yes, it was, so it had two numbers. I will multiply these two numbers with each } \\
\text { other and then multiply them with } 1 \text {. So, we will have width, length, and height. }\end{array}$ \\
\hline $\begin{array}{c}\vdots \\
\text { Item } 131\end{array}$ & Teacher & $\begin{array}{l}\text { : Did anyone use woodblocks? I saw someone brought it. Well, how you use these } \\
\text { woodblocks to do anything? }\end{array}$ \\
\hline Item 133 & All students & : We used it as a width $\mathrm{x}$ length $\mathrm{x}$ height formula. \\
\hline
\end{tabular}

There were two pieces of evidence of the fourth category of reasoning habits identified to be reflecting on a solution', such as observation artifact Item 125 Student 2 explained about 'Yes it was, so it had two numbers. I 
will multiply these two numbers with each other and then multiply them with 1. So we will have width, length, and height". The mentioned Item showed that students approved by answering and showing their reasoning, and from Item 133 "We used it as width $x$ length $x$ height formula", students were able to verify and conclude problemsolving by using a formula, which was the common problem-solving. These shreds of evidence were regarded as 'reflecting on the solution' which is the fourth category of students' reasoning habit.

\section{Discussion}

This research investigated $6^{\text {th }}$ Grade students' reasoning habits development after participating in LS and OA innovations by providing pieces of evidence based on NCTM's (2009) framework as a benchmark. The observation results revealed that students were able to describe their concepts, through previous knowledge and adapted with problem-solving methods. Besides, students could anticipate the way to solve problems, find various strategies, as well as connect different matters to solve problems by themselves, including able to prove and interpret the outcomes with reasonability. The results are found to support Oslington et al.'s (2020) research findings as mathematical reasoning is important to develop critical thinking and understand mathematics in a more meaningful way. The concepts of reasoning not only assist the students to have a deeper understanding of the subject but also assist them in having a wider perspective to the logical statement (Mathnasium of Littleton, 2016).

Moreover, the results of this research contribute to our acknowledgment of the prominence of LS and OA teaching practices in enhancing students' mathematical reasoning habits, namely analyzing a problem, implementing a strategy, seeking and using connections, and reflecting on a solution. The results further imply that the innovation of LS and OA is relevant to be an academic management model in educational institutions, as mentioned by Inprasitha (2016). Besides, one of the significant contributions to mathematics education is the interaction with students in a teaching experiment, the students' and teacher's actions are co-dependent. The realization that the researchers are participants in the students' constructions and the students are active participants in the researcher's constructions is precisely what recommends the teaching experiment methodology that is LS and OA innovations. In conclusion, the results can be rationalized in terms of the current evidence and should be applied to improve students' reasoning habits in the future.

\section{Acknowledgments}

The authors gratefully acknowledge use of service and facilities of the Centre for Research in Mathematics Education (CRME), Khon Kaen University, Khon Kaen 40002, Thailand. The contents of this manuscript are derived from the first author's doctoral dissertation thus fulfilling the PhD requirement of Khon Kaen University.

\section{References}

Becker, J. P., \& Shimada, S. (1997). The open-ended approach: A new proposal for teaching mathematics. Reston, VA: National Council of Teachers of Mathematics.

Boyd, P., \& Ash, A. (2018). Mastery mathematics: Changing teacher beliefs around in-class grouping and mindset. Teaching and Teacher Education, 75, 214-223. https://doi.org/10.1016/j.tate.2018.06.016

Eddy, C. M., \& Inprasitha, M. (2012). US-Thailand research network to advance the teaching and learning of algebraic reasoning research proposal. Khon Kaen, Thailand: Khon Kaen University.

Inprasitha, M. (2011). One feature of adaptive lesson study in Thailand: Designing a learning unit. Journal of Science and Mathematics Education in Southeast Asia, 34(1), 47-66.

Inprasitha, M. (2014). Processes of problem solving in school mathematics. Center for Research in Mathematics Education (CRME), Faculty of Education, Khon Kaen University, Thailand: Pen printing. [in Thai]

Inprasitha, M. (2016). A report on the synthesis of academic management models in educational institutions using the innovation of lesson study and open approach. Khon Kaen, Thailand: Klangnanavittaya Press.

Kaur, A., Awang Hashim, R., \& Noman, M. (2014). Teacher autonomy support: Does it hinder motivation among Thai student? Malaysian Journal of Learning and Instruction, 11, 271-189.

Mathnasium of Littleton. (2016). News from Mathnasium of Littleton: Understanding mathematical reasoning. Retrieved from https://www.mathnasium.com/littleton-news-understanding-mathematical-reasoning.

Meyer, R. D., \& Wilkerson, T. L. (2011). Lesson study: The impact on teachers' knowledge for teaching mathematics. In L. C. Hart, A. S. Alston, \& Murata (Eds.), Lesson study research and practice in mathematics education (pp. 15-26). London, UK: Springer Science + Business Media. https://doi.org/10.1007/978-90-4819941-9 2

National Council of Teachers of Mathematics (NCTM). (2009). Focus in high school mathematics reasoning and 
sense making. Reston, VA: National Council of Teachers of Mathematics.

Nohda, N. (2000). Teaching by open-approach method in Japanese mathematics classroom. Proceedings of the Conference of the International Group for the Psychology of Mathematics Education (PME), Hiroshima, Japan, July 23-27, 1, ERIC ED 466736.

Oslington, G., Mulligan, J., \& Van Bergen, P. (2020). Third-graders' predictive reasoning strategies. Educational Studies in Mathematics, 104, 5-24. https://doi.org/10.1007/s10649-020-09949-0

Steffe, L. P., \& Thompson, P. W. (2000). Teaching experiment methodology: Underlying principles and essential elements. In R. Lesh \& A. E. Kelly (Eds), Research design in mathematics and science education (pp. 267307). Hillsdale, NJ: Erlbaum.

Tall, D. (2008). Using Japanese lesson study in teaching mathematics. The Scottish Mathematical Council Journal, $38,45-50$.

Tan, S. F., Lim, C. S., \& Chew, C. M. (2017). Changes in teachers' reflection after lesson study process. Malaysian Journal of Learning and Instruction, Special Issue, 145-172.

Wang-Iverson, P., \& Yoshida, M. (Eds.). (2005). Building our understanding of lesson study. Philadelphia, PA: Research for Better Schools.

\section{Copyrights}

Copyright for this article is retained by the author(s), with first publication rights granted to the journal.

This is an open-access article distributed under the terms and conditions of the Creative Commons Attribution license (http://creativecommons.org/licenses/by/4.0/). 